\title{
Multimedia News Web Sites: A Cross- Cultural Analysis of Online Journalism in China and the UK
}

Ye Hao, Shanghai Jiao Tong University, China Guy Starkey, Bournemouth University, UK 


\section{Introducing... my research partner}

Dr Hao Ye, Shanghai Jiao Tong University, China

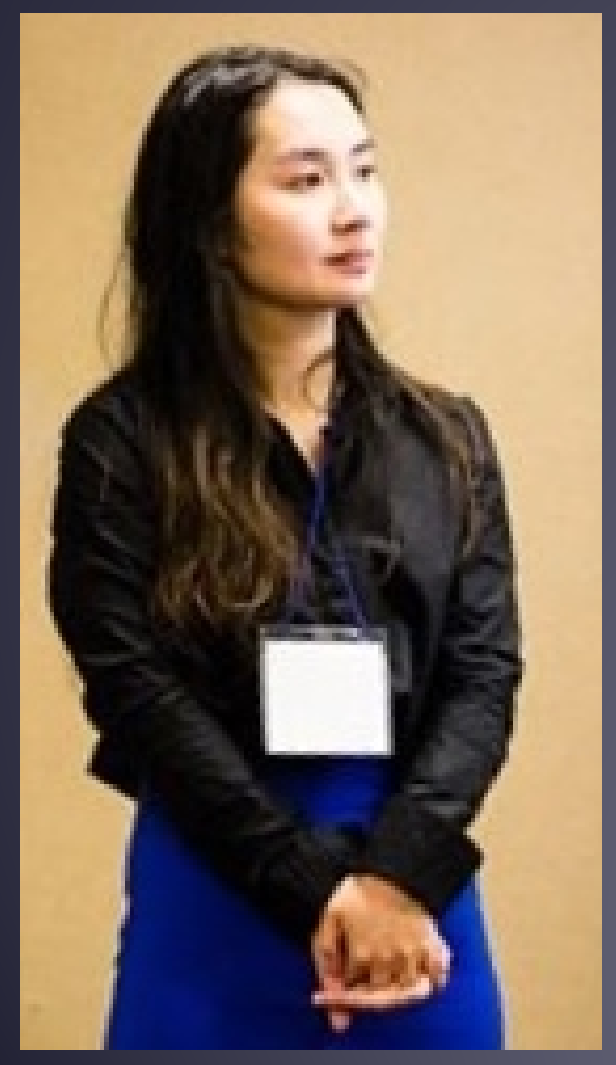

Undergraduate degree in journalism MA University of Leicester in mass communication

PhD University of Leicester, involving detailed quallitative and quantitative textual comparison between China and UK television advertising Currently teaching multimedia journalism techniques in China 


\section{Cross-cultural researchers 跨文化研究人员}

Dr Hao Ye, Shanghai Jiao Tong University, China Professor Guy Starkey, Bournemouth University, UK

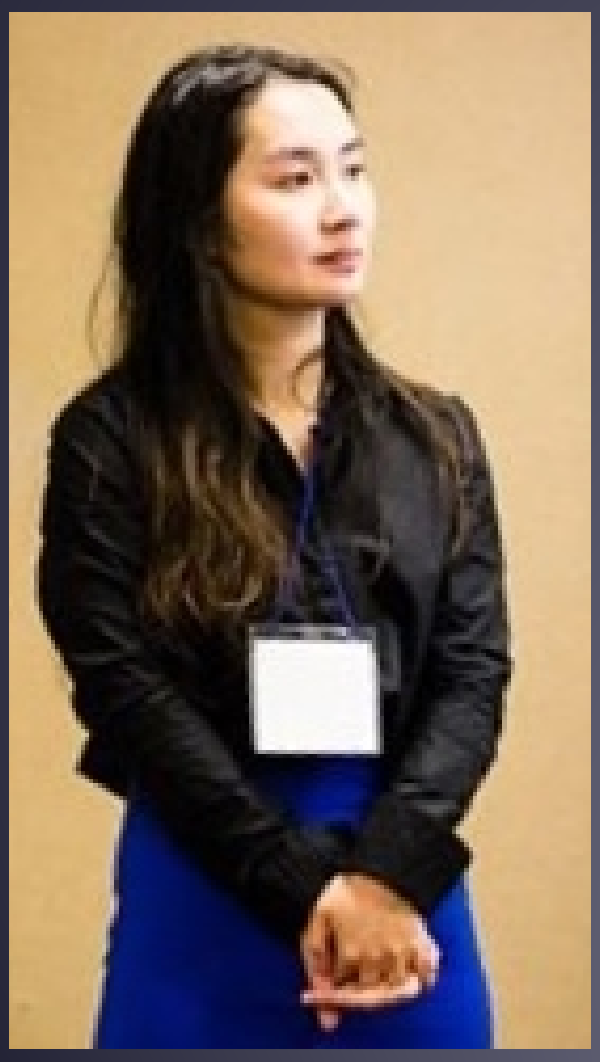

Shanghai Jiao Tong

- $104^{\text {th }}$ QS worldranked university

- School of Media \& Design Bournemouth

- 4 QS stars

- Top 200 subject QS ranking

- Faculty of Media \& Communication

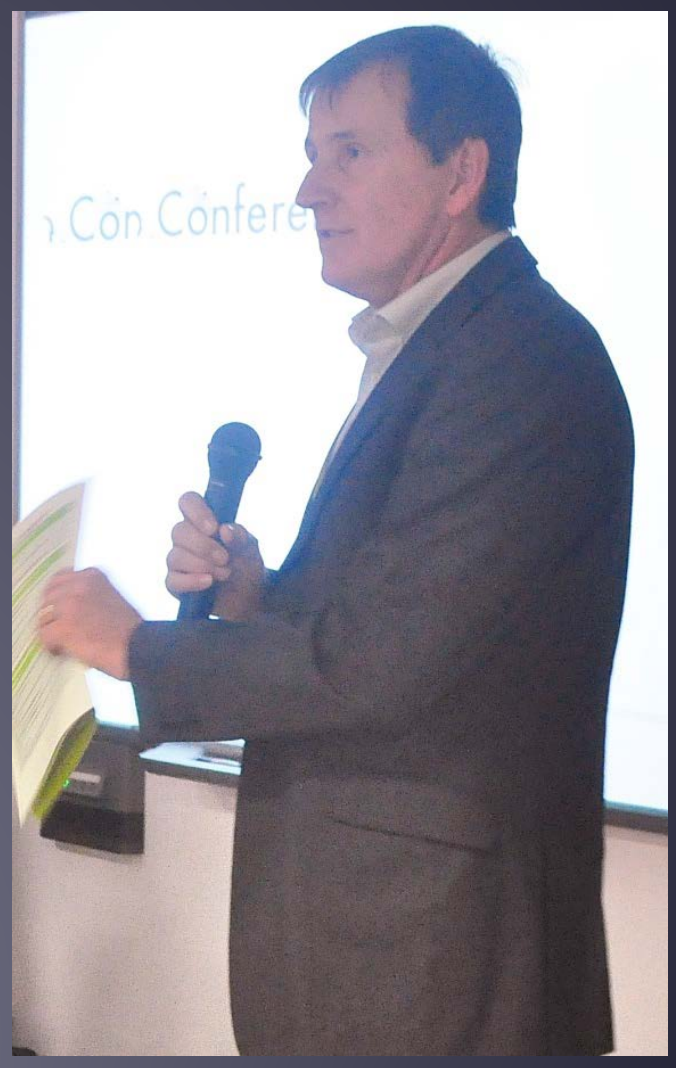




\section{Objectives of the project}

- To investigate the use of multimedia elements in online news journalism in the UK and China

- To identify synergies and harmonization across national and cultural divides
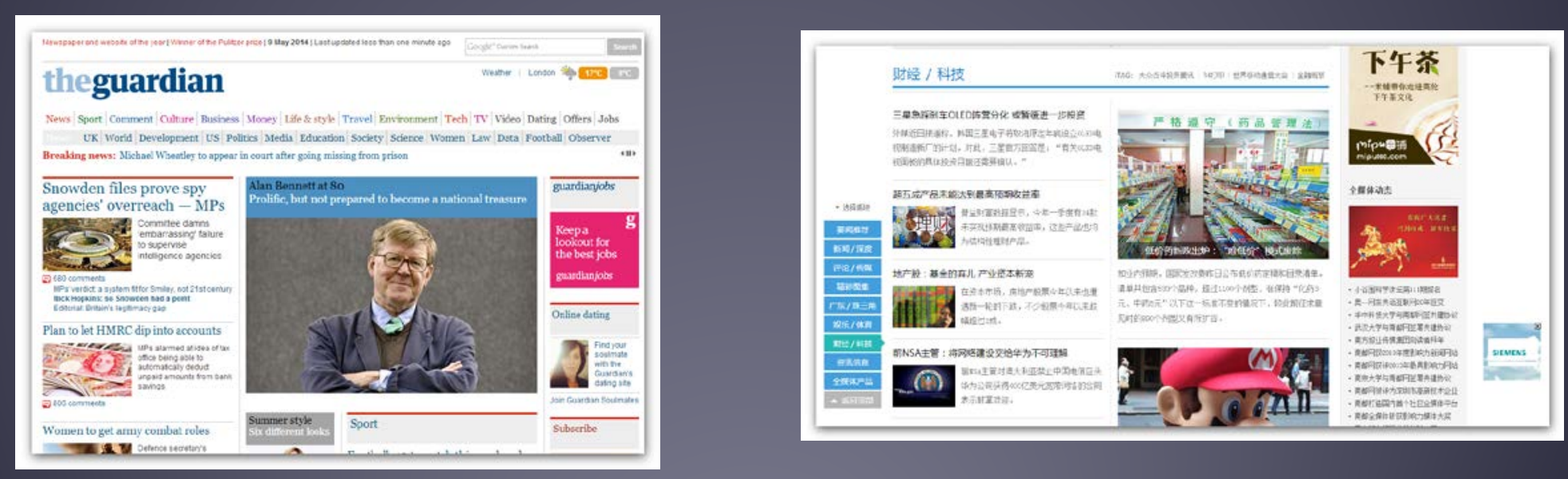


\section{The websites}

China

UK

- 新华网 Xinhua Wang

- BBC News

- 南都网 Nandu Wang

- The Guardian

- 人民网 Renming Wang

- The Telegraph

The 2014 sample dates

9 May 9 June 9 July 


\section{May}

- 新华网

The Central Discipline Inspection Commission of China invited Chinese netizens to expose party members breaching the discipline online

- 南都网

A man imitating police tricked three women into bearing children for him, and one of the children was sold

- 人民网

A speech from Xi Jinping 'we cannot use the formalism to oppose the formalism'
- BBC News

MPs raise concerns over tax collecting powers to take unpaid tax from bank accounts

- The Guardian Snowden files prove spy agencies' overreach , according to committee of MPs

- The Telegraph Taxman has power to raid your bank accounts 


\section{June}

- 新华网

2014 national college entrance examination reveals positive signs of education reforms

\section{- 南都网}

China investigating the abolition or amendment of 'asylum education'

- 人民网

Peoples' Daily 'We should keep high alert for traps of westernstyle democracy'
- BBC News

Schools inspector Ofsted to publish reports into schools infiltrated by 'Trojan' muslims

- The Guardian Karachi airport: Pakistani Taliban claim responsibility

- The Telegraph

David Cameron is missing part of case for Scotland to remain in UK 


\section{July}

- 新华网

- Xin Jingping: 'China and the United States should cooperate and avoid confrontation to benefit the whole world'

- 南都网

Police claimed 'Bus fire was arson, motive of the attack is still unknown'

- 人民网

Xi Jinping discusses Chinese and American relations 'We should not be puzzled by an incident, and not be harassed by a word'
- BBC News

National Society for the Prevention of Cruelty to Children wants law on negligence over covering up child abuse

- The Guardian

Israel peps up offensive against Gaza as missiles target Tel Aviv

- The Telegraph

Savvy shoppers force down retail prices of goods by shopping around 


\section{ND)南都网}

北京 $8^{\circ} \mathrm{C} \sim-3^{\circ} \mathrm{C}$

2014-5-9 星期五 花历四月十一

\section{登录 今日 邮件 南都 南都网 \\ 注册报纸 阅读官微群 手机版} 2014-5-9 星期五

(新闻 时局 国际 社会 深度 传媒 文化 数字报

评论 图片 视频 娱乐 体育 公益科技 财经

$\square$ 汽车 消费

$2 \begin{aligned} & \text { 空间 感光度 商城 } \\ & \text { 米捕 自媒体 动漫 }\end{aligned}$
( ) 广东

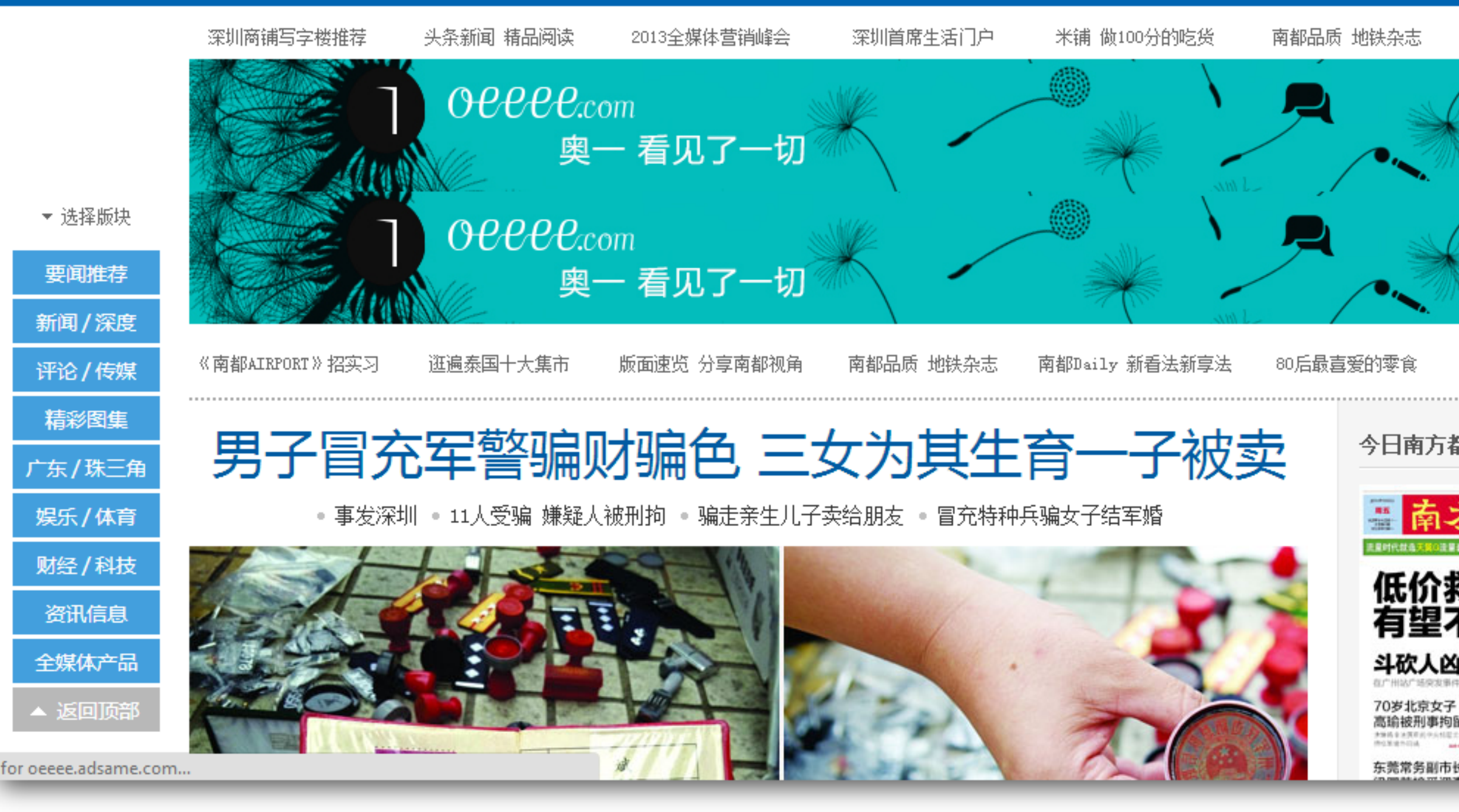


Apple to pay \$2bn for Dr Dre's Beats

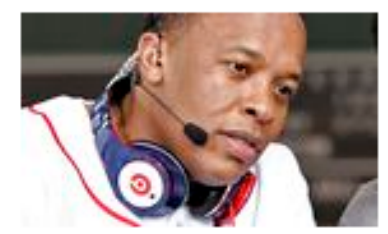

Reports suggest any deal could be worth $£ 1.88$ bn, the California company's largest ever acquisition

目 318 comments

\section{Half of students feel addicted to net}

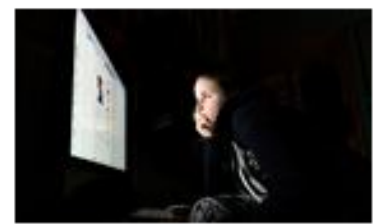

Survey finds $50 \%$ of year 10 students believe they are hooked, while threequarters take laptop, phone or tablet to bed

国 83 comments

EU considers meat slaughter labels

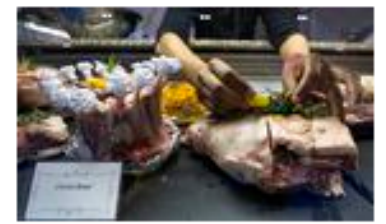

EU considering change backed by religious leaders but not by David Cameron - to bring clarity to industry

\section{日) 224 comments}

What does halal slaughter involve?

\section{Oscar Pistorius defence continues}

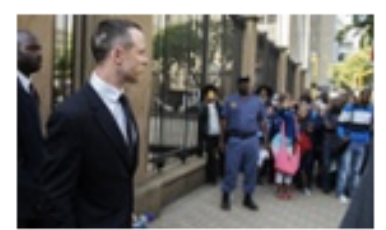

LIVE Live coverage from the athlete's murder trial as ballistics expert gives evidence for the defence

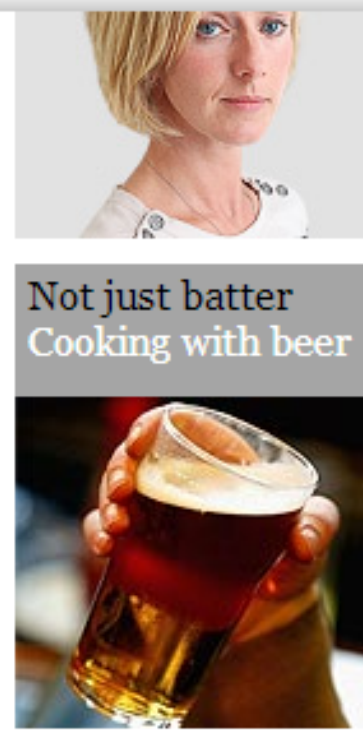

Potter: the play Rowling on board

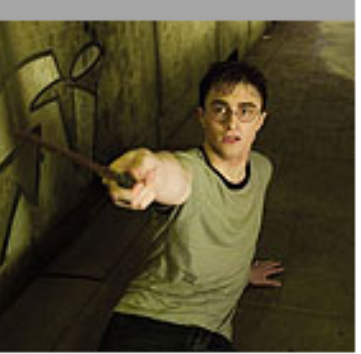

Graham Norton The $£ 2.3 \mathrm{~m}$ man

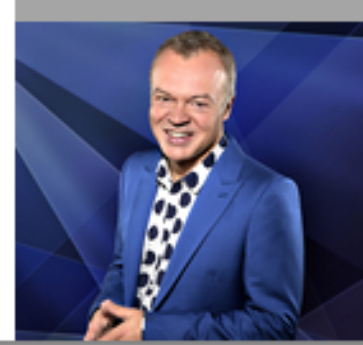

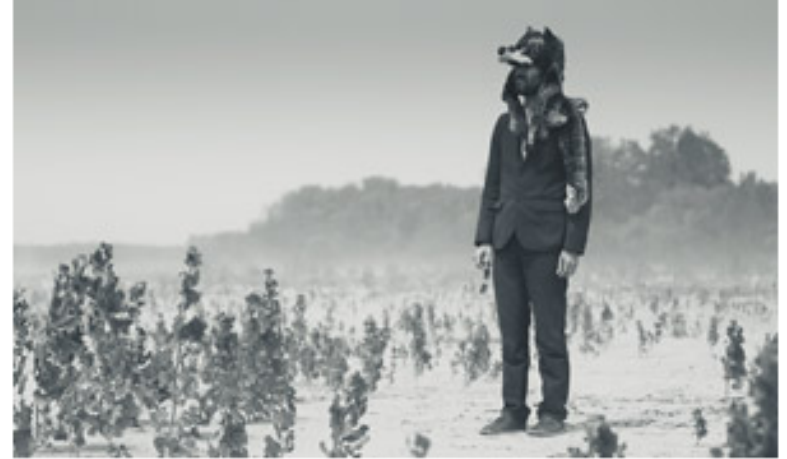

Gruff Rhys's American Interior

Super Furry Animal explains his 'tragicomic piece of history that is really sad and beautiful and damaged 国 15 comments

Alexis Petridis on American Interior

Fiona Shaw's five-star conjuring act

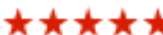

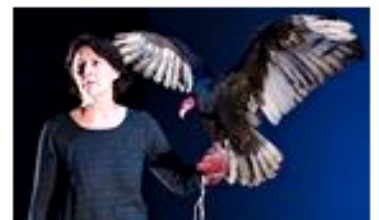

An ingenious and powerful adaptation of Colm Tóibín's novella The Testament of Mary, writes Michael Billington

目 12 comments

Lyn Gardner: where are the original new plays?

Noah: the three books of the film

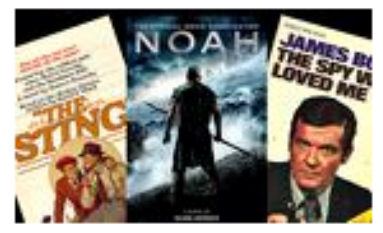

Darren Aronofsky's biblica special effects blockbuster has three incarnations in old-fashioned print, but novels spawned by films

国 37 comments
2014 Liverpool

Contemporary Arts fair

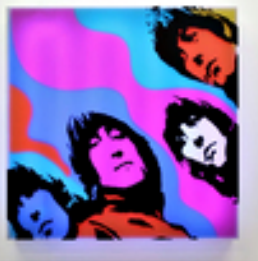

You could win travel, a night in a hotel and VIP tickets to the biggest arts show in the North.

Click here to find out more.

\section{More Extra offers}

Today's paper

Main section

G2 features

Comment and debate

Editorials, letters and corrections

Obituaries

Other lives

Sport

Film \& music 
河南新安房管局开发楼盘 局长 : 不搞房地产没饭吃

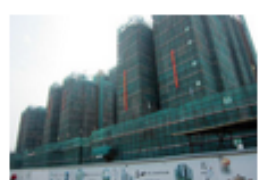

房地产公司认为, 公司根据上级批准成 立, 并一直合法经营, 解决房管局全体 职工的工资问题, 并无不篓。

沂蒙山万斤供品煎饼遭游客抢空 原定送福利院孩子

一选择版块

要闻推存

新闻 / 深度

评论 / 传媒

精彩图集

广东/珠三角

娱乐/体育

财经/科技

盗讯信息

全媒体产品

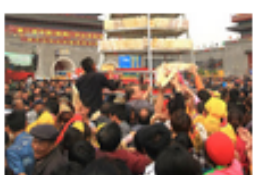

祈福仪式一开始就有游客不顾劝阻抢夺 这些作为供品的煎垪，随后短短十多分 钟, 近万斤煎饼就被哄抢一空。

\section{新疆阿克苏市处置一起䒾警案 嫌疑人投掷爆燃装置}

民警在对一嫌疑车辆盘查时, 光罪嫌疑人突然持刀袭警, 并 向巡逻车投挷爆燃装置。民警果断处置, 击䃈1人，抓获1 人。处置过程中造成一名协警重伤。

70 岁高瑜涉嫌泄露中央机密文件到境外 已经被刑拘

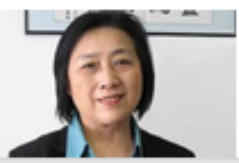

归案后, 高瑜表达深刻忓悔, 称甘愿接 受法律惩处。在1993年, 她曾因泄露国 家机密罪被判外有期徒刑哖

不用注册, 用这些帐号直接登录南都网 $\rightarrow 6$ 微博帐号

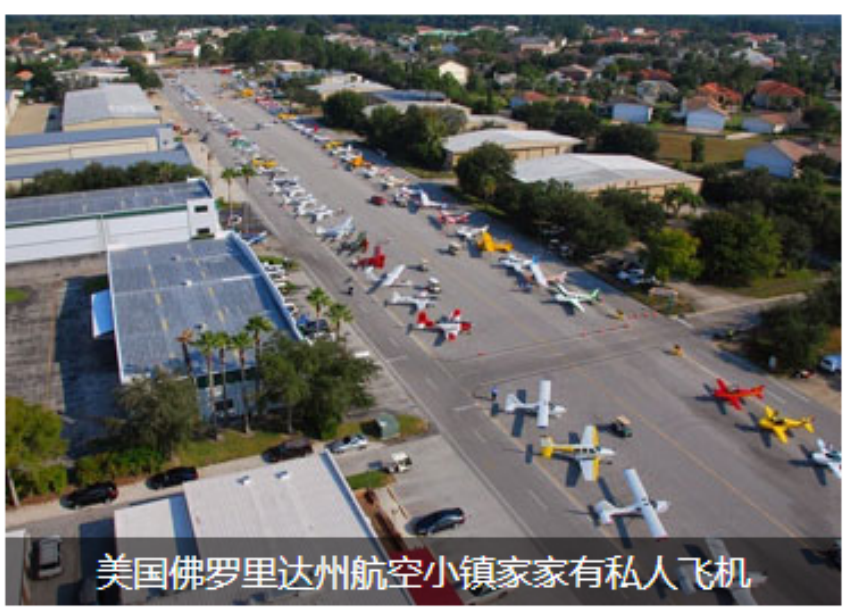

在美国佛罗里达州一片别墅住宅区内, 坐落着风格各异的房 屋, 而每一栋房屋门前都停放着1架或1架以上的飞机。房屋 门前的大道整齐宽阔, 并直通毗邻小区的一条修莀完整的飞 机跑道, 跑道上不时地会有飞机起飞或降落。
LA

- 培育和踐行社会主义核心价值观

- 图片策划: 雅安, 这一年

- 五彩广东 奥式精彩

- 奥生活 奥精彩

- 315策划: 互联网时代怎能被坑

- 特别策划: 两会数图汇

- 2014全国两会

- 南方都市报年终作品集

- 2014广州两会

- 2014广东两会

-2014春运

- 图刊: 马上有年终奖

- 南都网体育策划: 角力2013

- 南都网科技策划: 疯跑2013

- 南都网图片策划: 见证2013

- 寻找2013广东最美街坊

- 众望2013 · 深圳民生实事评选

- 古石阳: 垃圾在革我们的命

- 聚焦十八届三中全会

- 记者与他们的微言平台

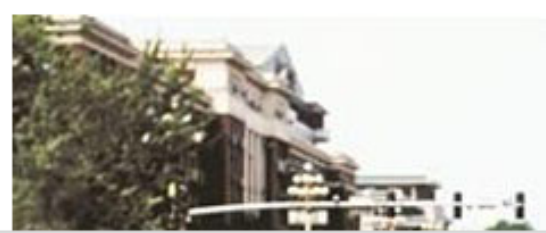

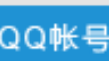

P 腾讯微榑胀号
南都网空间公测啦, 进入体验吧!

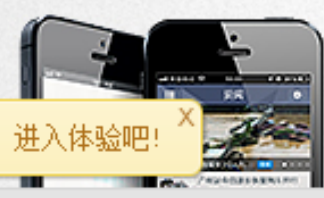

南都网空间》 


\section{News front page structure}

\begin{tabular}{|l|l|l|l|l|l|l|l|}
\hline China & $\begin{array}{l}\text { Scrolling } \\
\text { ticker }\end{array}$ & $\begin{array}{l}\text { Refresh } \\
\text { ing } \\
\text { ticker }\end{array}$ & $\begin{array}{l}\text { Display } \\
\text { advertisi } \\
\text { ng }\end{array}$ & UK & $\begin{array}{l}\text { Scrolli } \\
\text { ng } \\
\text { ticker }\end{array}$ & $\begin{array}{l}\text { Refresh } \\
\text { ing } \\
\text { ticker }\end{array}$ & $\begin{array}{l}\text { Display } \\
\text { advertisi } \\
\text { ng }\end{array}$ \\
\hline $\begin{array}{l}\text { Xinhua } \\
\text { She }\end{array}$ & $\mathrm{Y}$ & $\mathrm{N}$ & $\mathrm{Y}$ & BBC News & $\mathrm{N}$ & $\mathrm{Y}$ & $\mathrm{N}$ \\
\hline $\begin{array}{l}\text { Nandu } \\
\text { Wang }\end{array}$ & $\mathrm{N}$ & $\mathrm{N}$ & $\mathrm{Y}$ & $\begin{array}{l}\text { The } \\
\text { Guardian }\end{array}$ & $\mathrm{N}$ & $\mathrm{Y}$ & $\mathrm{Y}$ \\
\hline $\begin{array}{l}\text { Renming } \\
\text { Wang }\end{array}$ & $\mathrm{N}$ & $\mathrm{N}$ & $\mathrm{Y}$ & $\begin{array}{l}\text { The } \\
\text { Telegraph }\end{array}$ & $\mathrm{N}$ & $\mathrm{N}$ & $\mathrm{Y}$ \\
\hline
\end{tabular}


Streams and feature pages

\begin{tabular}{|c|c|c|c|c|c|c|c|}
\hline China & $\begin{array}{l}\text { live } \\
\text { radio } \\
\text { stream }\end{array}$ & $\begin{array}{l}\text { live TV } \\
\text { stream }\end{array}$ & $\begin{array}{l}\text { feature } \\
\text { pages }\end{array}$ & UK & $\begin{array}{l}\text { live } \\
\text { radio } \\
\text { stream }\end{array}$ & $\begin{array}{l}\text { live TV } \\
\text { stream }\end{array}$ & $\begin{array}{l}\text { feature } \\
\text { pages }\end{array}$ \\
\hline $\begin{array}{l}\text { Xinhua } \\
\text { She }\end{array}$ & & $\begin{array}{l}9 \text { (XINHUA } \\
\text { TV) }\end{array}$ & $\begin{array}{l}764 \\
678 \\
528\end{array}$ & BBC News & $\begin{array}{l}1 \text { (BBC } \\
\text { Radio } 5 \\
\text { Live) }\end{array}$ & $\begin{array}{l}1 \text { (BBC } \\
\text { News } \\
\text { Channel) }\end{array}$ & $\begin{array}{l}15 \\
4 \\
15\end{array}$ \\
\hline $\begin{array}{l}\text { Nandu } \\
\text { Wang }\end{array}$ & & & $\begin{array}{l}43 \\
46 \\
57 \\
\end{array}$ & $\begin{array}{l}\text { The } \\
\text { Guardian }\end{array}$ & & & $\begin{array}{l}65 \\
45 \\
\end{array}$ \\
\hline $\begin{array}{l}\text { Renming } \\
\text { Wang }\end{array}$ & & $\begin{array}{l}14 \text { (RMTV) } \\
14 \\
\text { (Renming } \\
\text { TV) } \\
12 \text { (RMTV) }\end{array}$ & $\begin{array}{l}281 \\
331 \\
345\end{array}$ & $\begin{array}{l}\text { The } \\
\text { Telegraph }\end{array}$ & & & $\begin{array}{l}4 \\
7\end{array}$ \\
\hline
\end{tabular}


Number of stories featured on the news front pages of the six sites surveyed, subdivided into international and national

\begin{tabular}{|l|l|l|l|l|l|}
\hline China & International & National & UK & International & National \\
\hline $\begin{array}{l}\text { Xinhua } \\
\text { She }\end{array}$ & 5 & 100 & BBC News & 9 & 27 \\
& 4 & 92 & & 12 & 12 \\
22 & & 37 \\
\hline $\begin{array}{l}\text { Nandu } \\
\text { Wang }\end{array}$ & 3 & 63 & The & 12 & 11 \\
& 1 & 61 & Guardian & 7 & 18 \\
Renming & 6 & 61 & & 11 & 15 \\
Wang & 2 & 59 & The & 8 & 22 \\
& 3 & 68 & Telegraph & 7 & 24 \\
& & 51 & & 7 & 24 \\
\hline
\end{tabular}


Number of news front page stories with visible links to multimedia content

\begin{tabular}{|l|l|l|l|l|l|l|l|}
\hline China & $\begin{array}{l}\text { Any } \\
\text { multimed } \\
\text { ia } \\
\text { content }\end{array}$ & $\begin{array}{l}\text { Video } \\
\text { content }\end{array}$ & $\begin{array}{l}\text { Audio } \\
\text { content }\end{array}$ & UK & $\begin{array}{l}\text { Any } \\
\text { multim } \\
\text { edia } \\
\text { conten } \\
\text { t }\end{array}$ & $\begin{array}{l}\text { Video } \\
\text { content }\end{array}$ & $\begin{array}{l}\text { Audio } \\
\text { content }\end{array}$ \\
\hline $\begin{array}{l}\text { Xinhua } \\
\text { She }\end{array}$ & 4 & 4 & 0 & BBC News & 11 & 10 & 1 \\
& 2 & 4 & 0 & & 9 & 9 & 0 \\
\hline Nandu & 0 & 0 & 0 & The & 0 & 0 & 0 \\
Wang & 0 & 0 & 0 & Guardian & 1 & 1 & \\
\hline Renming & 0 & 0 & 0 & & 1 & 1 & 0 \\
\hline Wang & 0 & 0 & 0 & The & 5 & 5 & 0 \\
& 0 & 0 & 0 & Telegraph & 3 & 3 & 0 \\
\hline
\end{tabular}




\section{Conclusions}

- China multimedia news websites have three times as many stories as the UK sites

- Both China and UK focus on national news at the expense of international stories, which are mainly lower down the page or in specialised sections

- UK sites feature more international stories than the Chinese sites

- Both China and UK multimedia news websites use mainly text and still image to present content, with relatively very little use of video and audio elements to illustrate stories 


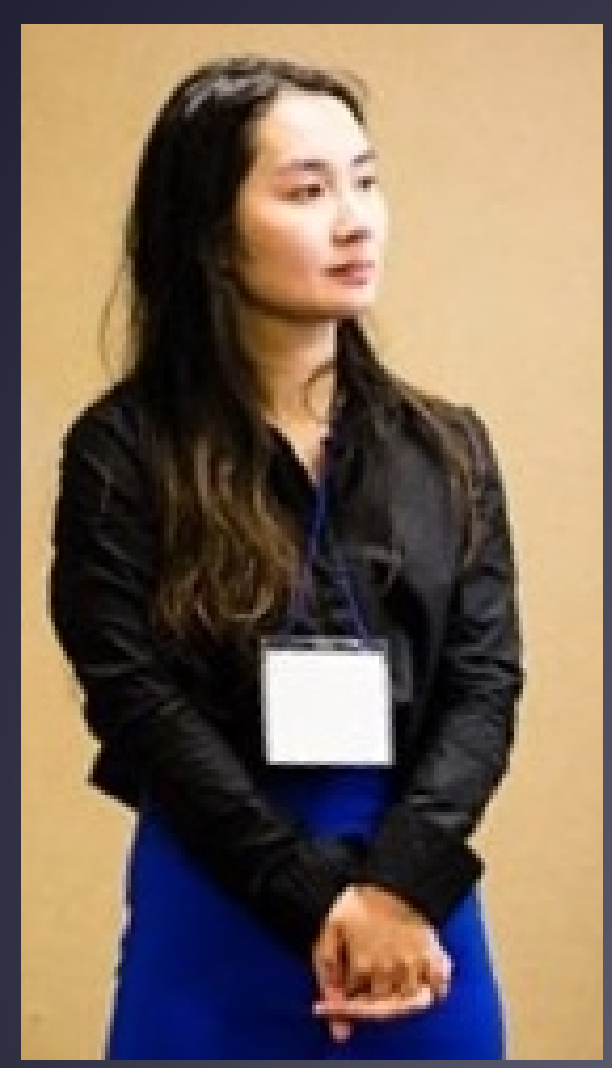

Thank

you for

listening!

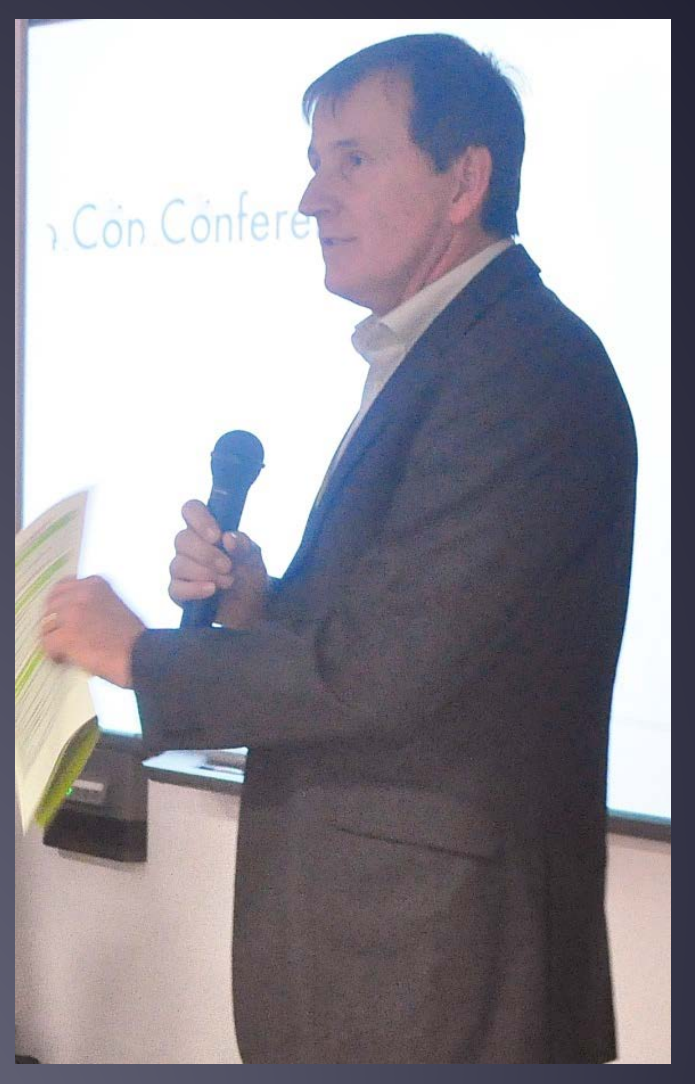

